\title{
Clinical Correlations Between Cesium, Cobalt, Manganese, Rubidium and Rhenium with the Pathogenesis of Rheumatoid Arthritis
}

\author{
Shatha Rouf Moustafa \\ Clinical Analysis Department, College of Pharmacy, Hawler Medical University, Havalan City, Erbil, Iraq
}

Email address:

shatha003@yahoo.com

To cite this article:

Shatha Rouf Moustafa. Clinical Correlations Between Cesium, Cobalt, Manganese, Rubidium and Rhenium with the Pathogenesis of Rheumatoid Arthritis. Science Journal of Clinical Medicine. Vol. 4, No. 5, 2015, pp. 99-108. doi: 10.11648/j.sjcm.20150405.15

\begin{abstract}
Background and Objectives: Rheumatoid arthritis is one of the most common inflammatory diseases. Studies indicated that increased oxidative stress or defective antioxidant status with inflammation contribute to the pathogenesis of disease. This study was emphasized that levels of some trace elements altered due to oxidative stress and inflammatory process which are associated with the pathogenesis of rheumatoid arthritis. This study was aimed to assess the serum levels of cesium, cobalt, manganese, rubidium and rhenium in patients group as compared with the control group and investigate the effect of other confounding factors like rheumatoid factor, age, gender and finally estimate the correlation between studied parameters. It was hypothesized that there were an alteration in levels of parameters due to oxidative stress and inflammatory process which are associated with the etiology and pathogenesis of rheumatoid arthritis. Method: This case control study conducted on 56 Iraqi patients of both genders 30 men and 26 women with the average age (40-57) years and 56 of age -gender matched apparently healthy adults were also enrolled in this study as a control group. The patients were free from any diseases except rheumatoid arthritis and this was confirmed by clinical examination and biochemical and hematological tests. Levels of parameters were estimated using atomic absorption spectrophotometers. Results: There were no significant differences between RF positive and negative groups regarding the studied parameters. There were a significant reduction in cesium and rubidium and a significant elevations in cobalt, manganese and rhenium in patients as compared with the control group. There were no significant differences between rheumatoid factor positive and negative groups regarding focused parameters in men and women groups, except for age factor in women group, there were no significant differences between different age categories in rheumatoid factor positive and negative regarding focused parameters. In addition, there was no significant correlation among studied parameters. Conclusion: Levels of parameters were significantly altered in patients group. These alterations proved the possible association with pathogenesis of rheumatoid arthritis. Therefore, the estimation of these parameters could be a useful complementary non-invasive diagnostic tool to determine trace elements status for treatment and diagnosis of disease. These variations are probably responsive to oxidative stress concomitant with inflammation induced by cytokines and resulted from defensive mechanism of patients. Inflammatory mediators affect on trace elements homeostasis in rheumatoid arthritis.
\end{abstract}

Keywords: Rheumatoid Arthritis, Cesium, Cobalt, Manganese, Rubidium and Rhenium

\section{Introduction}

\subsection{General View}

Rheumatoid arthritis (RA) is a chronic, inflammatory and systemic disease of unknown etiology. Although the cause of RA is still unknown, much points towards a multi-factorial etiology such as environmental factors and genetic factors which play a role in the development and progression of the
RA [1].

Inflammation and oxidative stress (OS) have been implicated in the pathogenesis of rheumatoid arthritis [2].

Rheumatoid arthritis is characterized by increased activity of macrophages, which in cooperation with other inflammatory cells infiltrates the synovial tissue. The activated macrophages, monocytes and granulocytes generate reactive oxygen species (ROS) which have been suggested to be mediators of inflammation, together with the pro- 
inflammatory cytokines.

It has been suggested that ROS and trace elements (TEs) play a role in the etiology and pathogenesis of rheumatoid arthritis (RA). Deficiencies of TEs in patients with RA occur in response to chronic inflammation.

There is a shift toward oxidant/antioxidant imbalance favor to lipid peroxidation, which could lead to the tissue damage. Elevated free radicals production in inflamed joints and dysfunction of antioxidant system have been implicated in RA [3]. Many of these TEs are incorporated into antioxidant enzymes.

The increased levels of inflammatory mediators like cytokines such as interleukin-1 (IL-1), tumor necrosis factor$\alpha$ (TNF- $\alpha$ ) and IL- 6 are associated with active RA which may affect the bioavailability of these TEs by stimulation the production of metal-binding proteins in the liver or intestine. The increased production of metallothionein may affect the level of metal ions so that they are deficient in blood circulation.

Physiological concentration of ROS may be beneficial in process such as intracellular signaling and defense against micro-organsim. Nevertheless, higher amounts of ROS play a role in the aging process as well as in a number of human diseases including inflammatory state, rheumatoid arthritis, cancer, failure in immunity and endocrine functions.

However, many studies showed that there was an association between TEs status with RA.

Trace elements are enrolled in many important processes associated to health, structural support, contraction of muscle, enzyme / hormones release nerve conduction, and maintenance of mineral balance in human body [4].

The importance of the TEs in RA is of great interest because many of these TEs are co-factors in metabolic processes involving collagen and bone structure, articular tissues or immune system functions [5].

Trace elements may play a role in the initiation, progression and curing of the disease as investigated by supplying different TEs to decrease pain and increase joint mobility [6].

\subsection{Cesium (Cs)}

Caesium is known as cesium in the USA. Cesium is one of the most alkaline elements and raises the $\mathrm{pH}$ of the body's cells.

This metal is characterized by a spectrum containing two bright lines in the blue (accounting for its name). It is silvery gold, soft, and ductile. It is the most electropositive and most alkaline element. It is liquid at or around room temperature which reacts explosively with cold water, and reacts with ice above $-116^{\circ} \mathrm{C}$. Cesium hydroxide is a strong base and attacks glass.

Maintaining proper body tissue $\mathrm{pH}$ is critical for staying healthy. Alkaline tissue holds 20 times more oxygen than does acidic tissue and this oxygen rich environment is critical for supporting health.

Cesium has been found to be capable of significantly raising the $\mathrm{pH}$ of malignant cancer cells to the point of the cells dying off-usually within days without harming normal cells.
Cesium not only kills cancer (even in stage 4 patients), but also relieves pain by neutralizing the lactic acid that cancer makes from fermenting sugar for energy. The cells that die are absorbed and eliminated by the body.

Cesium salts may be useful in the treatment of manicdepressives. It is used to supports the body's immune system and regulates cardiac rhythm.

\subsection{Cobalt (Co)}

Cobalt is valuable in cases of headaches, migraines, vasomotor disorders, vertigo and tinnitus. This is due to the sympathicolytic effect that Co exerts on vascular receptors. Cobalt has a regulating influence on stomach and bowel receptors Cobalt, and other heavy metals are essential TEs, but they are toxic when their concentrations are relatively high. Possible mechanisms of action of these metals might include denaturation of protein, inhibition of enzymes, or increased susceptibility to infection due to immunosuppression. Metal exposuremay occur also by contamination of food and drinking water.

Several researchers have reported that the ingestion of one or more heavy elements may be important in the etiology of some rheumatic diseases like RA.

Cobalt is required as a constituent of vitamin B12 and its metabolism is the same as for vitamin B12, Co is also a cofactor of enzymes involved in amino acid metabolism and DNA biosynthesis [7]. It acts as a co-factor in antioxidant therapy for the enzyme catalase. It is essential for the growth and development of healthy nervous system.

Deficiencies are reflected in abnormal bone development [8]. It is also necessary co-factor for making the thyroid hormone and deficiency lead to slow growth and development conditions [8], 30\% of the population are deficient in Co and could benefit from Co supplementation.

Because of its low retention rate, Co toxicity is rare, but an excess can lead to enlargement of the thyroidgland.

\subsection{Manganese (Mn)}

Manganese is one of TEs that are necessary for bone health, 36-90\% of the population are deficient in $\mathrm{Mn}$ and could benefit from Mn supplementation. Manganese deficiency can lead to abnormal bone formation. An excess of Mn affect iron metabolism (poor iron metabolism) [9].

It is an essential part of many enzymes associated with protein and fat metabolism, energy production and bone formation, it is also required for the utilization of vitamin $\mathrm{E}$ and vitamin B1, formation of cartilage and synovial fluid of the joints. Manganese is biochemically essential as an enzyme activator [9], synthesis of acid mucopolysaccharidessuch as chondroitin sulphatewhich is utilized to form collagen, bone and cartilage, strong fibrous connective material that builds tissue. Manganese is involved in glycoprotein synthesis and it is a co-factor in phosphohydrolases and phosphotransferases involved in the synthesis of proteoglycans in cartilage. Consequently skeletal deformities occurs when the Mn intake is notsufficient [10]. 
Manganese is important in mental facilities and functions. It aids memory and modulates neurotransmitter activity related to nerve/muscle dysfunction.

Manganese is a part of enzymes involved in urea formation, pyruvate metabolism and the galactotransferase of connective tissue biosynthesis [11].

Carpal Tunnel Syndrome, a painful condition in which arm tendons are weak or damaged, is a sign of manganese deficiency.

Deafness, if due to damage of the cartilage of the ear, can be attributed partially to a deficiency in Mn.

Manganese is stored half in the bones and the remainder in the liver, pancreas, pituitary gland, and the kidneys.

A deficiency of Mn concentration may affect brain health and skeletal and cartilage formation, it is a constituent of metalloenzymes antioxidant enzyme superoxide dismutase which prevents damage by superoxide free radicals [12].

It is used for therapy of RA, allergies, arthritis, fatigue, headache, migraine, inflammation, thyroid disorders, respiratory problems, sinusitis, sinus infection.

\subsection{Rhenium $(\operatorname{Re})$}

Rhenium is silvery white element. It is expensive but useful as a trace alloying agent.

${ }^{186} \mathrm{Re}$ sulphide colloid offers a local, relatively noninvasive therapeutic technique for the treatment of arthritic joint conditions in medium-sized joints such as thehip and ankle, shoulder, elbow and wrist.

\subsection{Rubidium (Rb)}

Rubidium is a soft, silvery white metallic element. It is used in some laboratory studiesand clinical applications. Rubidium stimulates metabolism.

Rubidium chloride is used to induce cells to take up DNA, Rbions are taken up by cells of living organisms similar to potassium ions to replace potassium that is taken up by animal cells and plant. It has a spectral absorption range, making it a target for laser manipulation of atoms. As a result of variation in the blood brain barrier in brain tumors, brain tumors have a higher concentration of Rbthan normal brain tissue, allowing the use of rubidium- 82 in nuclear medicine to locate and image brain tumors [13].

Depressed patients show deficient in $\mathrm{Rb}$ concentration, so supplementation may be necessary for those patients [14].It is used in high $\mathrm{PH}$ therapy and to decrease tumor growth,muscle pain, multiple sclerosis, tetany, spasmophilia. Depletion of $\mathrm{Rb}$ affects minerals (zinc, copper, phosphorus, calcium, magnesium, iron, sodium and potassium) status.

Accordingly, the aim of the present study was undertaken to assess the probable variations in the serum levels of $\mathrm{Ce}$, $\mathrm{Co}, \mathrm{Mn}, \mathrm{Rb}$ and $\mathrm{Rh}$ in patients with $\mathrm{RA}$. In addition, to investigate their relationship with other confounding factors RF, age, gender and finally evaluate the correlation coefficient between all studied parameters.

It was hypothesized that there were an alteration in serum profile of interested parameters due to OS concomitant with inflammatory process which are associated with the etiology and pathogenesis of RA.

\section{Patients and Methods}

This study was performed to assess the association between the serum level of focused parameters with the pathogenesis of RA. This study was done at Hawler Medical University, College of Pharmacy in period between 2014 and2015.

\subsection{Study Design}

This case control study conducted on 56 Iraqi patients of both genders 30 men (16 men with rheumatoid factor positive and 14 men with rheumatoid factor negative) and 26 women (12 women with rheumatoid factor positive and 14 women with rheumatoid factor negative) with the average age (40-57) years, in addition an equal number of apparently healthy adults randomly selected were also participated in this study as a control group. Serum trace elements were determined using Atomic Absorption Spectrophotometer in Baghdad city at Ministry of Science and Technology /Department of Research of Biochemical Science. The protocol of the study was approved by Ethical Committee of College of Pharmacy. Verbal consent was obtained from all subjects before participation.

Samples were collected under the guidance of expert rheumatologist. All reagents and chemicals were of high analytical grade. Exclusion criteria: None of the patients and controls were smokers or consuming alcohol or had any other chronic disease except RA and this is confirmed by clinical examination and laboratory estimations of biochemical and hematological tests.

\subsection{Sample Collection}

The Protocol of the Study: Ten $\mathrm{ml}$ of the fasting blood (overnight for 12-14 hours) samples was collected from the vein of the participants (healthy adults and patients with RA) of both genders without using tourniquet. The blood samples were left for 30 minutes for coagulation purpose, and then centrifuged for 15 minutes at 2500-3500 revolution per minutes (rpm). The sera of the patients were separated and divided into several parts and put them into several plastic plain tubes to do the biochemical tests of the current study.

The sera of the patients werestoredat $\left(-80 \mathrm{C}^{\circ}\right)$ till the day of the analysis within (1-2 months). The sera samples were prepared for measurement by warming the frozen sera at room temperature.

\subsection{Statistical Analysis}

The SPSS 18.0 software was used for statistical analysis. Statistical significance was defined as $P<0.05$.

\section{Results}

The patient samples were divided into two groups rheumatoid factor (RF) positive and RF negative 
groups. Thedemographic distribution of patients was shown in (Table1).

Table 1. The demographic distribution of patients group.

\begin{tabular}{llllllll}
\hline Gender & \multicolumn{4}{l}{ Rheumatoid factor } & \multicolumn{2}{l}{ Total } & p \\
\hline & \multicolumn{2}{l}{ Negative } & \multicolumn{2}{l}{ Positive } & & & \\
\hline Male & 14 & 46.7 & 16 & 53.3 & 30 & 100 & 0.592 \\
Female & 14 & 53.8 & 12 & 46.2 & 26 & 100 & \\
Total & 28 & 50 & 28 & 50 & 56 & 100 & \\
\hline
\end{tabular}

There were no significant differences between RF positive and negative groups regarding the studied parameters (Table 2).

Accordingly, there was no RF effect on the serum levels of studied parameters.

Table 2. Comparison between $R F$ positive and negative groups regarding the studied parameters.

\begin{tabular}{llllll}
\hline & RF & N & Mean & \pm SD & p \\
\hline \multirow{2}{*}{ Age } & Negative & 28 & 49.18 & 4.78 & .20 \\
& Positive & 28 & 46.21 & 4.95 & .027 \\
$\mathrm{Co}$ & Negative & 28 & .07 & .01 & .214 \\
& Positive & 28 & .07 & .01 & \\
$\mathrm{Mn}$ & Negative & 28 & .03 & .00 & .457 \\
& Positive & 28 & .03 & .00 & \\
$\mathrm{Ce}$ & Negative & 28 & 23.31 & 3.68 & .981 \\
& Positive & 28 & 23.34 & 3.75 & \\
$\mathrm{Rb}$ & Negative & 28 & 22.78 & 3.85 & .099 \\
& Positive & 28 & 24.38 & 3.24 & \\
$\mathrm{Rh}$ & Negative & 28 & 28.21 & 5.09 & .889 \\
& Positive & 28 & 28.39 & 4.37 & \\
\hline
\end{tabular}

Table 4. Comparison between different age categories the regarding studied parameters in RF negative group

\begin{tabular}{|c|c|c|c|c|c|c|c|}
\hline \multirow{2}{*}{ Dependent Variable } & \multirow{2}{*}{ (I) Age_Cat } & \multirow{2}{*}{ (J) Age_Cat } & \multirow{2}{*}{ Mean Difference (I-J) } & \multirow{2}{*}{ Std. Error } & \multirow{2}{*}{ Sig. } & \multicolumn{2}{|c|}{ 95\% Confidence Interval } \\
\hline & & & & & & Lower Bound & Upper Bound \\
\hline \multirow{11}{*}{ Co } & \multirow{3}{*}{$40-44$} & $45-49$ & .003433 & .005179 & .514 & -.00725 & .01412 \\
\hline & & $50-54$ & -.003036 & .004613 & .517 & -.01256 & .00648 \\
\hline & & $55+$ & .000433 & .005179 & .934 & -.01025 & .01112 \\
\hline & \multirow{3}{*}{$45-49$} & $40-44$ & -.003433 & .005179 & .514 & -.01412 & .00725 \\
\hline & & $50-54$ & -.006470 & .004340 & .149 & -.01543 & .00249 \\
\hline & & $55+$ & -.003000 & .004938 & .549 & -.01319 & .00719 \\
\hline & \multirow{3}{*}{$50-54$} & $40-44$ & .003036 & .004613 & .517 & -.00648 & .01256 \\
\hline & & $45-49$ & .006470 & .004340 & .149 & -.00249 & .01543 \\
\hline & & $55+$ & .003470 & .004340 & .432 & -.00549 & .01243 \\
\hline & \multirow{3}{*}{$55+$} & $40-44$ & -.000433 & .005179 & .934 & -.01112 & .01025 \\
\hline & & $45-49$ & .003000 & .004938 & .549 & -.00719 & .01319 \\
\hline \multirow{13}{*}{$\mathrm{Mn}$} & & $50-54$ & -.003470 & .004340 & .432 & -.01243 & .00549 \\
\hline & \multirow{3}{*}{$40-44$} & $45-49$ & .004600 & .002379 & .066 & -.00032 & .00952 \\
\hline & & $50-54$ & .003655 & .002029 & .085 & -.00054 & .00785 \\
\hline & & $55+$ & $.007033^{*}$ & .002278 & .005 & .00232 & .01175 \\
\hline & \multirow{3}{*}{$45-49$} & $40-44$ & -.004600 & .002379 & .066 & -.00952 & .00032 \\
\hline & & $50-54$ & -.000945 & .002029 & .646 & -.00514 & .00325 \\
\hline & & $55+$ & .002433 & .002278 & .297 & -.00228 & .00715 \\
\hline & \multirow{3}{*}{$50-54$} & $40-44$ & -.003655 & .002029 & .085 & -.00785 & .00054 \\
\hline & & $45-49$ & .000945 & .002029 & .646 & -.00325 & .00514 \\
\hline & & $55+$ & .003379 & .001909 & .090 & -.00057 & .00733 \\
\hline & \multirow{3}{*}{$55+$} & $40-44$ & $-.007033 *$ & .002278 & .005 & -.01175 & -.00232 \\
\hline & & $45-49$ & -.002433 & .002278 & .297 & -.00715 & .00228 \\
\hline & & $50-54$ & -.003379 & .001909 & .090 & -.00733 & .00057 \\
\hline \multirow{5}{*}{$\mathrm{Ce}$} & \multirow{3}{*}{$40-44$} & $45-49$ & -2.98400 & 2.15612 & .179 & -7.4340 & 1.4660 \\
\hline & & $50-54$ & -2.83945 & 1.92050 & .152 & -6.8032 & 1.1243 \\
\hline & & $55+$ & .23433 & 2.15612 & .914 & -4.2157 & 4.6843 \\
\hline & \multirow{2}{*}{$45-49$} & $40-44$ & 2.98400 & 2.15612 & .179 & -1.4660 & 7.4340 \\
\hline & & $50-54$ & .14455 & 1.80713 & .937 & -3.5852 & 3.8743 \\
\hline
\end{tabular}

Table 3. Comparison between patient and control groups regarding studied parameters in $R F$ positive and negative groups.

\begin{tabular}{llll}
\hline & Patients group ng/ml & Control group ng/ml & P value \\
\hline Cs & & & \\
RF- & $23.3107 \pm 3.678$ & $30 \pm 4.3$ & 0.001 \\
RF+ & $23.3350 \pm 3.749$ & & \\
Co & & & \\
RF- & $0.06596 \pm 0.008$ & $0.035 \pm 0.001$ & 0.001 \\
RF+ & $0.06942 \pm 0.011$ & & \\
Mn & & & \\
RF- & $0.03130 \pm 0.004$ & $0.025 \pm 0.003$ & 0.001 \\
RF+ & $0.03042 \pm 0.004$ & & \\
Re & & & \\
RF- & $28.21 \pm 5.087$ & $25 \pm 3.47$ & \\
RF+ & $28.39 \pm 4.374$ & & 0.001 \\
Rb & & & \\
RF- & $22.7836 \pm 3.846$ & $35 \pm 2.9$ & \\
RF+ & $24.3764 \pm 3.236$ & & \\
\hline
\end{tabular}

There were significant differences between patient and control groups regarding the studied parameters. There were significant elevations in the serum levels of $\mathrm{Co}, \mathrm{Mn}, \mathrm{Re}$ and $\mathrm{Rb}$ in patients group as compared with the control group. Moreover, there were significant reduction in the serum levels of $\mathrm{Cs}$ and $\mathrm{Rb}$ in patients group as compared with the control group (Table 3 ).

There were no significant differences between different age categories regarding all studied parametersin $\mathrm{RF}$ negative group (Table 4) except for $\mathrm{Mn}(\mathrm{age}=55+, \mathrm{p}=0.005)$ and $\mathrm{Rb}$ ( age $=40-44, \mathrm{p}=0.044)$. 


\begin{tabular}{|c|c|c|c|c|c|c|c|}
\hline \multirow{14}{*}{ Dependent Variable } & \multirow{2}{*}{ (I) Age_Cat } & \multirow{2}{*}{ (J) Age_Cat } & \multirow{2}{*}{ Mean Difference (I-J) } & \multirow{2}{*}{ Std. Error } & \multirow{2}{*}{ Sig. } & \multicolumn{2}{|c|}{$95 \%$ Confidence Interval } \\
\hline & & & & & & Lower Bound & Upper Bound \\
\hline & & $55+$ & 3.21833 & 2.05578 & .131 & -1.0246 & 7.4612 \\
\hline & & $40-44$ & 2.83945 & 1.92050 & .152 & -1.1243 & 6.8032 \\
\hline & $50-54$ & $45-49$ & -.14455 & 1.80713 & .937 & -3.8743 & 3.5852 \\
\hline & & $55+$ & 3.07379 & 1.80713 & .102 & -.6559 & 6.8035 \\
\hline & & $40-44$ & -.23433 & 2.15612 & .914 & -4.6843 & 4.2157 \\
\hline & $55+$ & $45-49$ & -3.21833 & 2.05578 & .131 & -7.4612 & 1.0246 \\
\hline & & $50-54$ & -3.07379 & 1.80713 & .102 & -6.8035 & .6559 \\
\hline & & $45-49$ & $-4.80967 *$ & 2.25786 & .044 & -9.4697 & -.1497 \\
\hline & $40-44$ & $50-54$ & -2.84709 & 2.01112 & .170 & -6.9978 & 1.3037 \\
\hline & & $55+$ & -2.01800 & 2.25786 & .380 & -6.6780 & 2.6420 \\
\hline & & $40-44$ & $4.80967 *$ & 2.25786 & .044 & .1497 & 9.4697 \\
\hline & $45-49$ & $50-54$ & 1.96258 & 1.89240 & .310 & -1.9431 & 5.8683 \\
\hline \multirow{12}{*}{$\mathrm{Rb}$} & & $55+$ & 2.79167 & 2.15278 & .207 & -1.6515 & 7.2348 \\
\hline & & $40-44$ & 2.84709 & 2.01112 & .170 & -1.3037 & 6.9978 \\
\hline & $50-54$ & $45-49$ & -1.96258 & 1.89240 & .310 & -5.8683 & 1.9431 \\
\hline & & $55+$ & .82909 & 1.89240 & .665 & -3.0766 & 4.7348 \\
\hline & & $40-44$ & 2.01800 & 2.25786 & .380 & -2.6420 & 6.6780 \\
\hline & $55+$ & $45-49$ & -2.79167 & 2.15278 & .207 & -7.2348 & 1.6515 \\
\hline & & $50-54$ & -.82909 & 1.89240 & .665 & -4.7348 & 3.0766 \\
\hline & & $45-49$ & .933 & 3.229 & .775 & -5.73 & 7.60 \\
\hline & $40-44$ & $50-54$ & 1.964 & 2.876 & .501 & -3.97 & 7.90 \\
\hline & & $55+$ & 1.933 & 3.229 & .555 & -4.73 & 8.60 \\
\hline & & $40-44$ & -.933 & 3.229 & .775 & -7.60 & 5.73 \\
\hline & $45-49$ & $50-54$ & 1.030 & 2.706 & .707 & -4.56 & 6.62 \\
\hline \multirow{7}{*}{$\mathrm{Rh}$} & & $55+$ & 1.000 & 3.079 & .748 & -5.35 & 7.35 \\
\hline & & $40-44$ & -1.964 & 2.876 & .501 & -7.90 & 3.97 \\
\hline & $50-54$ & $45-49$ & -1.030 & 2.706 & .707 & -6.62 & 4.56 \\
\hline & & $55+$ & -.030 & 2.706 & .991 & -5.62 & 5.56 \\
\hline & & $40-44$ & -1.933 & 3.229 & .555 & -8.60 & 4.73 \\
\hline & $55+$ & $45-49$ & -1.000 & 3.079 & .748 & -7.35 & 5.35 \\
\hline & & $50-54$ & .030 & 2.706 & .991 & -5.56 & 5.62 \\
\hline
\end{tabular}

*. The mean difference is significant at the 0.05 level.

There were no significant differences between different age categories regarding the studied parameters. Accordingly, there was no age effect, in RF positive group. (Table 5).

Table 5. Comparison between different age categories the regarding studied parameters in $R F$ positive group.

\begin{tabular}{|c|c|c|c|c|c|c|c|}
\hline \multirow{2}{*}{$\begin{array}{l}\text { Dependent } \\
\text { Variable }\end{array}$} & \multirow{2}{*}{ (I) Age_Cat } & \multirow{2}{*}{ (J) Age_Cat } & \multirow{2}{*}{ Mean Difference (I-J) } & \multirow{2}{*}{ Std. Error } & \multirow{2}{*}{ Sig. } & \multicolumn{2}{|c|}{ 95\% Confidence Interval } \\
\hline & & & & & & Lower Bound & Upper Bound \\
\hline \multirow{12}{*}{ Co } & \multirow{3}{*}{$40-44$} & $45-49$ & .003750 & .006774 & .585 & -.01030 & .01780 \\
\hline & & $50-54$ & -.000667 & .005830 & .910 & -.01276 & .01142 \\
\hline & & $55+$ & -.004500 & .009033 & .623 & -.02323 & .01423 \\
\hline & \multirow{3}{*}{$45-49$} & $40-44$ & -.003750 & .006774 & .585 & -.01780 & .01030 \\
\hline & & $50-54$ & -.004417 & .007713 & .573 & -.02041 & .01158 \\
\hline & & $55+$ & -.008250 & .010348 & .434 & -.02971 & .01321 \\
\hline & \multirow{3}{*}{$50-54$} & $40-44$ & .000667 & .005830 & .910 & -.01142 & .01276 \\
\hline & & $45-49$ & .004417 & .007713 & .573 & -.01158 & .02041 \\
\hline & & $55+$ & -.003833 & .009756 & .698 & -.02407 & .01640 \\
\hline & \multirow{3}{*}{$55+$} & $40-44$ & .004500 & .009033 & .623 & -.01423 & .02323 \\
\hline & & $45-49$ & .008250 & .010348 & .434 & -.01321 & .02971 \\
\hline & & $50-54$ & .003833 & .009756 & .698 & -.01640 & .02407 \\
\hline \multirow{12}{*}{$\mathrm{Mn}$} & \multirow{3}{*}{$40-44$} & $45-49$ & -.003071 & .002315 & .198 & -.00787 & .00173 \\
\hline & & $50-54$ & .001429 & .001993 & .481 & -.00270 & .00556 \\
\hline & & $55+$ & -.004571 & .003087 & .153 & -.01097 & .00183 \\
\hline & \multirow{3}{*}{$45-49$} & $40-44$ & .003071 & .002315 & .198 & -.00173 & .00787 \\
\hline & & $50-54$ & .004500 & .002636 & .102 & -.00097 & .00997 \\
\hline & & $55+$ & -.001500 & .003537 & .676 & -.00883 & .00583 \\
\hline & \multirow{3}{*}{$50-54$} & $40-44$ & -.001429 & .001993 & .481 & -.00556 & .00270 \\
\hline & & $45-49$ & -.004500 & .002636 & .102 & -.00997 & .00097 \\
\hline & & $55+$ & -.006000 & .003335 & .086 & -.01292 & .00092 \\
\hline & \multirow{3}{*}{$55+$} & $40-44$ & .004571 & .003087 & .153 & -.00183 & .01097 \\
\hline & & $45-49$ & .001500 & .003537 & .676 & -.00583 & .00883 \\
\hline & & $50-54$ & .006000 & .003335 & .086 & -.00092 & .01292 \\
\hline
\end{tabular}




\begin{tabular}{|c|c|c|c|c|c|c|c|}
\hline \multirow{2}{*}{$\begin{array}{l}\text { Dependent } \\
\text { Variable }\end{array}$} & \multirow{2}{*}{ (I) Age_Cat } & \multirow{2}{*}{ (J) Age_Cat } & \multirow{2}{*}{ Mean Difference (I-J) } & \multirow{2}{*}{ Std. Error } & \multirow{2}{*}{ Sig. } & \multicolumn{2}{|c|}{ 95\% Confidence Interval } \\
\hline & & & & & & Lower Bound & Upper Bound \\
\hline \multirow{13}{*}{$\mathrm{Ce}$} & \multirow{3}{*}{$40-44$} & $45-49$ & 1.30536 & 2.12097 & .544 & -3.0721 & 5.6828 \\
\hline & & $50-54$ & -2.04214 & 1.65803 & .230 & -5.4642 & 1.3799 \\
\hline & & $55+$ & 1.67786 & 2.82795 & .559 & -4.1588 & 7.5145 \\
\hline & \multirow{3}{*}{$45-49$} & $40-44$ & -1.30536 & 2.12097 & .544 & -5.6828 & 3.0721 \\
\hline & & $50-54$ & -3.34750 & 2.29090 & .157 & -8.0757 & 1.3807 \\
\hline & & $55+$ & .37250 & 3.23983 & .909 & -6.3142 & 7.0592 \\
\hline & \multirow{3}{*}{$50-54$} & $40-44$ & 2.04214 & 1.65803 & .230 & -1.3799 & 5.4642 \\
\hline & & $45-49$ & 3.34750 & 2.29090 & .157 & -1.3807 & 8.0757 \\
\hline & & $55+$ & 3.72000 & 2.95754 & .221 & -2.3841 & 9.8241 \\
\hline & \multirow{3}{*}{$55+$} & $40-44$ & -1.67786 & 2.82795 & .559 & -7.5145 & 4.1588 \\
\hline & & $45-49$ & -.37250 & 3.23983 & .909 & -7.0592 & 6.3142 \\
\hline & & $50-54$ & -3.72000 & 2.95754 & .221 & -9.8241 & 2.3841 \\
\hline & \multirow{3}{*}{$40-44$} & $45-49$ & -1.79607 & 1.79779 & .328 & -5.5065 & 1.9144 \\
\hline \multirow{11}{*}{$\mathrm{Rb}$} & & $50-54$ & -.91357 & 1.40539 & .522 & -3.8142 & 1.9870 \\
\hline & & $55+$ & 3.46643 & 2.39705 & .161 & -1.4808 & 8.4137 \\
\hline & \multirow{3}{*}{$45-49$} & $40-44$ & 1.79607 & 1.79779 & .328 & -1.9144 & 5.5065 \\
\hline & & $50-54$ & .88250 & 1.94183 & .654 & -3.1252 & 4.8902 \\
\hline & & $55+$ & 5.26250 & 2.74616 & .067 & -.4053 & 10.9303 \\
\hline & \multirow{3}{*}{$50-54$} & $40-44$ & .91357 & 1.40539 & .522 & -1.9870 & 3.8142 \\
\hline & & $45-49$ & -.88250 & 1.94183 & .654 & -4.8902 & 3.1252 \\
\hline & & $55+$ & 4.38000 & 2.50689 & .093 & -.7940 & 9.5540 \\
\hline & \multirow{3}{*}{$55+$} & $40-44$ & -3.46643 & 2.39705 & .161 & -8.4137 & 1.4808 \\
\hline & & $45-49$ & -5.26250 & 2.74616 & .067 & -10.9303 & .4053 \\
\hline & & $50-54$ & -4.38000 & 2.50689 & .093 & -9.5540 & .7940 \\
\hline \multirow{12}{*}{$\mathrm{Rh}$} & \multirow{3}{*}{$40-44$} & $45-49$ & -.143 & 2.207 & .949 & -4.70 & 4.41 \\
\hline & & $50-54$ & 3.232 & 1.725 & .073 & -.33 & 6.79 \\
\hline & & $55+$ & $-6.143^{*}$ & 2.942 & .048 & -12.22 & -.07 \\
\hline & \multirow{3}{*}{$45-49$} & $40-44$ & .143 & 2.207 & .949 & -4.41 & 4.70 \\
\hline & & $50-54$ & 3.375 & 2.384 & .170 & -1.54 & 8.29 \\
\hline & & $55+$ & -6.000 & 3.371 & .088 & -12.96 & .96 \\
\hline & \multirow{3}{*}{$50-54$} & $40-44$ & -3.232 & 1.725 & .073 & -6.79 & .33 \\
\hline & & $45-49$ & -3.375 & 2.384 & .170 & -8.29 & 1.54 \\
\hline & & $55+$ & $-9.375^{*}$ & 3.077 & .006 & -15.73 & -3.02 \\
\hline & \multirow{3}{*}{$55+$} & $40-44$ & $6.143 *$ & 2.942 & .048 & .07 & 12.22 \\
\hline & & $45-49$ & 6.000 & 3.371 & .088 & -.96 & 12.96 \\
\hline & & $50-54$ & $9.375^{*}$ & 3.077 & .006 & 3.02 & 15.73 \\
\hline
\end{tabular}

*. The mean difference is significant at the 0.05 level.

There were no significant differences between RF positive and negative groups in males group (Table 6).

Table 6. Comparison between RF positive and negative groups in males group.

\begin{tabular}{llllll}
\hline & RF & N & Mean & SD & p \\
\hline \multirow{2}{*}{ Age } & Negative & 14 & 49.14 & 4.72 & .235 \\
& Positive & 16 & 46.94 & 5.17 & .235 \\
Co & Negative & 14 & .07 & .01 & .538 \\
& Positive & 14 & .07 & .01 & \\
\multirow{2}{*}{$\mathrm{Mn}$} & Negative & 14 & .03 & .00 & .610 \\
& Positive & 14 & .03 & .00 & \\
$\mathrm{Ce}$ & Negative & 14 & 23.46 & 3.54 & .817 \\
& Positive & 16 & 23.14 & 3.95 & \\
$\mathrm{Rb}$ & Negative & 14 & 23.34 & 4.07 & .381 \\
& Positive & 16 & 24.56 & 3.41 & \\
$\mathrm{Rh}$ & Negative & 14 & 29.64 & 6.45 & .638 \\
& Positive & 16 & 28.63 & 5.26 & \\
\hline
\end{tabular}

a. Gender $=$ Male

There were no significant differences between RF positive and negative groups in females group (Table 7)
Table 7. Comparison between RF positive and negative groups in females group.

\begin{tabular}{llllll}
\hline & RF & N & Mean & SD & p \\
\hline \multirow{2}{*}{ Age } & Negative & 14 & 49.21 & 5.01 & .249 \\
& Positive & 12 & 45.25 & 4.69 & \\
$\mathrm{Co}$ & Negative & 14 & .07 & .01 & .235 \\
& Positive & 12 & .07 & .01 & \\
$\mathrm{Mn}$ & Negative & 13 & .03 & .00 & .563 \\
& Positive & 12 & .03 & .00 & \\
$\mathrm{Ce}$ & Negative & 14 & 23.16 & 3.94 & .773 \\
& Positive & 12 & 23.60 & 3.63 & \\
$\mathrm{Rb}$ & Negative & 14 & 22.22 & 3.68 & .170 \\
& Positive & 12 & 24.14 & 3.12 & \\
$\mathrm{Rh}$ & Negative & 14 & 26.79 & 2.78 & .264 \\
& Positive & 12 & 28.08 & 3.00 & \\
\hline
\end{tabular}

There were no significant correlations among the studied parameters in RF negative group, except the correlation between Mn and age ( $\mathrm{p}=0.013, \mathrm{r}=-0.472)$ (Table 8$)$. 
Table 8. Correlation coefficient among studied parameters in RF negative group.

\begin{tabular}{|c|c|c|c|c|c|c|c|}
\hline & & Co & Mn & $\mathrm{Ce}$ & $\mathbf{R b}$ & $\mathbf{R h}$ & Age \\
\hline \multirow{4}{*}{ Co } & Pearson Correlation & 1 & -.085 & .024 & -.235 & .268 & .010 \\
\hline & Sig. (2-tailed) & & .674 & .902 & .229 & .167 & .959 \\
\hline & $\mathrm{N}$ & 28 & 28 & 28 & 28 & 28 & 28 \\
\hline & Pearson Correlation & -.085 & 1 & .084 & .099 & -.284 & $-.472^{*}$ \\
\hline \multirow[t]{3}{*}{$\mathrm{Mn}$} & Sig. (2-tailed) & .674 & & .678 & .624 & .150 & .013 \\
\hline & $\mathrm{N}$ & 28 & 28 & 28 & 28 & 28 & 28 \\
\hline & Pearson Correlation & .024 & .084 & 1 & .002 & -.343 & -.038 \\
\hline \multirow[t]{3}{*}{$\mathrm{Ce}$} & Sig. (2-tailed) & .902 & .678 & & .992 & .074 & .850 \\
\hline & $\mathrm{N}$ & 28 & 28 & 28 & 28 & 28 & 28 \\
\hline & Pearson Correlation & -.235 & .099 & .002 & 1 & -.155 & .141 \\
\hline \multirow[t]{3}{*}{$\mathrm{Rb}$} & Sig. (2-tailed) & .229 & .624 & .992 & & .430 & .475 \\
\hline & $\mathrm{N}$ & 28 & 28 & 28 & 28 & 28 & 28 \\
\hline & Pearson Correlation & .268 & -.284 & -.343 & -.155 & 1 & -.163 \\
\hline \multirow[t]{3}{*}{$\mathrm{Rh}$} & Sig. (2-tailed) & .167 & .150 & .074 & .430 & & .407 \\
\hline & $\mathrm{N}$ & 28 & 28 & 28 & 28 & 28 & 28 \\
\hline & Pearson Correlation & .010 & $-.472 *$ & -.038 & .141 & -.163 & 1 \\
\hline \multirow[t]{2}{*}{ Age } & Sig. (2-tailed) & .959 & .013 & .850 & .475 & .407 & \\
\hline & $\mathrm{N}$ & 28 & 28 & 28 & 28 & 28 & 28 \\
\hline
\end{tabular}

There were no significant correlations among the studied parameters in RF positive group (Table 9).

Table 9. Correlation coefficient among studied parameters in RF positive group.

\begin{tabular}{|c|c|c|c|c|c|c|c|}
\hline & & Co & Mn & $\mathrm{Ce}$ & $\mathbf{R b}$ & $\mathbf{R h}$ & Age \\
\hline & Pearson Correlation & 1 & .222 & -.200 & -.019 & .079 & .018 \\
\hline \multirow[t]{3}{*}{ Co } & Sig. (2-tailed) & & 277 & .327 & .926 & .701 & .929 \\
\hline & $\mathrm{N}$ & 28 & 28 & 28 & 28 & 28 & 28 \\
\hline & Pearson Correlation & .222 & 1 & -.251 & -.082 & .163 & .100 \\
\hline \multirow[t]{3}{*}{$\mathrm{Mn}$} & Sig. (2-tailed) & 277 & & .216 & .692 & .427 & .626 \\
\hline & $\mathrm{N}$ & 28 & 28 & 28 & 28 & 28 & 28 \\
\hline & Pearson Correlation & -.200 & -.251 & 1 & -.036 & -.363 & .007 \\
\hline \multirow[t]{3}{*}{$\mathrm{Ce}$} & Sig. (2-tailed) & .327 & .216 & & .857 & .057 & .973 \\
\hline & $\mathrm{N}$ & 28 & 28 & 28 & 28 & 28 & 28 \\
\hline & Pearson Correlation & -.019 & -.082 & -.036 & 1 & -.075 & -.020 \\
\hline \multirow[t]{3}{*}{$\mathrm{Rb}$} & Sig. (2-tailed) & .926 & .692 & .857 & & .706 & .920 \\
\hline & $\mathrm{N}$ & 26 & 26 & 28 & 28 & 28 & 28 \\
\hline & Pearson Correlation & .079 & .163 & -.363 & -.075 & 1 & -.002 \\
\hline \multirow[t]{3}{*}{$\mathrm{Rh}$} & Sig. (2-tailed) & .701 & .427 & .057 & .706 & & .991 \\
\hline & $\mathrm{N}$ & 28 & 28 & 28 & 28 & 28 & 28 \\
\hline & Pearson Correlation & .018 & .100 & .007 & -.020 & -.002 & 1 \\
\hline \multirow[t]{2}{*}{ Age } & Sig. (2-tailed) & .929 & .626 & .973 & .920 & .991 & \\
\hline & $\mathrm{N}$ & 28 & 28 & 28 & 28 & 28 & 28 \\
\hline
\end{tabular}

a. $\mathrm{RF}=$ Positive

\section{Discussion}

\subsection{General View}

It has been suggested that oxidative stress (OS), inflammatory process and TEs play a role in the pathogenesis and etiology of rheumatoid arthritis (RA). Deficiencies of TEs in patients with RA occur in response to OS and chronic inflammation.

The role of these TEs in chronic inflammatory conditions such as RA is of great interest because many of them are cofactors in metabolic processes involving collagen and bone or immune system function.

In peripheral tissues, within inflammatory cells, many of these trace metallic elements are incorporated into antioxidant enzymes. Antioxidant metalloenzymes interfere with the production of free radicals by inactivating reactive oxygen molecules in tissues and immune cells. Although the physiologic role for these TEs may be reasonably well substantiated, their therapeutic usefulness is less compelling.

Inflammation concomitant with OS have been implicated in the pathogenesis of rheumatoidarthritis [2].

This study was undertaken to examine the association between the levels of some TEs with the pathogenesis of RA. It has been suggested that levels of some TEs altered due to OS concomitant with inflammatory process which are associated clinically with RA.

The aim of this study was to assess the association between the serum levels of interested elements in a sample of Iraqi patients with incidence of RA and to investigate their relationship with other confounding factors $\mathrm{RF}$, age, gender and finally evaluate the correlation coefficient between all 
studied parameters. So, this study was designed to investigate the probable changes in the levels of some TEs in RA.

In the present study, it was observed that RA was characterized by increased RF level which is a marker of inflammation. This proved the involvement of inflammation with incidence of RA. Rheumatoid factor was independent significant predictor of altered serum levels of interested parameters regarding RF positive group.

The demographic characteristics of the patients group was presented in (Table1).

The comparison between RF positive and RF negative groupswasshown in (Table2).

\subsection{The Effect of Rheumatoid Arthritis on the Serum Levels of Focused Parameters}

\subsubsection{Cesium}

The serum level of cesium was significantly decreased in patients group as compared with the control group (Table 3).

Cesium is alkaline elements and raises the $\mathrm{pH}$ of the body's cells.

Maintaining proper body $\mathrm{pH}$ is important for staying healthy. Alkaline tissue holds 20 times more oxygen than does acidic tissue and this oxygen rich environment is critical for supporting health, this fact is compatible with current result that there was a significant reduction in serum level of Cs in patients with RA. So, supplementation with cesium as a therapeutic measure must be considered.

The result of the current study was not consistent with the previous result [15] who reported that, the serum levels of cesium in patients with rheumatoid arthritis werehigh.

Since there was a significant reduction in serum levels of this parameter, so supplementation with this TE could be necessary for RA therapy or as complementary for treatment of RA and even more important to individuals who are at high risk of developing RA.Studies of the nutritional status of patients with active RA frequently demonstrate deficiencies in micronutrient.

The increase in levels of pro-inflammatory cytokines such as interleukin-1 (IL-1), tumor necrosis factor- $\alpha$ (TNF- $\alpha$ ) and IL- 6 seen in the setting of active RA may affect the availability of TEs by inducing the production of metal-binding proteins, metallothioneins, in the liver or intestine. The increased metallothionein production may result in sequestration of these metal ions so that they are deficient [16].

\subsubsection{Cobalt}

The serum level of cobalt was significantly elevated in patient group as compared with the control group (Table3).

The result of the current study was consistent with the previous result [17] While, the result of current study was not agreeable was with previous results [18],who reported that the mean serum levels of cobalt was significantly lower than control group.

There was no any information to explain this significant elevation in serum levels of Co in patients with RA in all previous researches that deal with the association of RA with Co element.

\subsubsection{Manganese}

The serum level of manganese was significantly elevated in patient group as compared with the control group (Table3).

The result of the current study was concordant with previous results $[15,18,19]$.

The explanation for this significant elevation, the scientific researches were carefully reviewed and detected, no any information about this variation in serum levels of $\mathrm{Mn}$ in patients with RA. So, need further researches to explain this significant elevation of $\mathrm{Mn}$.

\subsubsection{Rhenium}

The serum level of Re was significantly elevated in patient group as compared with the control group (Table 3 ).

The scientific researches were carefully reviewed and detected that there were no any previous results dealing with the association of RA with the serum levels of Re, accordingly, this research was the first study dealing with the changes in the serum levels of Re in patients as compared with control group.

The exact explanation for the significant elevation in the serum levels of Re was not clear and need further studies to explain this clinical correlation between serum levels of Re with RA.

\subsubsection{Rubidium}

There was a significant reduction in serum levels of rubidiumin patients group as compared with the control group (Table 3), so supplementation with this TE could be important for treatment of RA and even more necessary to individuals who are at high risk of developing RA.

These variations in the serum level of focused parameters may provide opportunity to the physician to diagnose individuals who are at high risk to develop RA for correcting these imbalance of these elements.

The variation of TEs in sera of patients with RA can enable us to fox and shade more light on the role of TEs in physiological and pathological conditions.

This findings proved the possible association between altered levels of interested TEs with RA.

It could be suggested that the deficiency of $\mathrm{Cs}$ and $\mathrm{Rb}$ might be used as complementary non-invasive parameters for early diagnosis and treatment of RA.

The scientific researches were carefully reviewed and detected that there were no any previous results dealing with the association of RA with the serum levels of $R b$, accordingly, this research was the first study dealing with the changes in the serum levels of $\mathrm{Rb}$ in patients with $\mathrm{RA}$ as compared with control group.

\subsection{Gender-Factor}

Rheumatoid arthritis is 2-3 times more common in women than in men. But in current study, there was no gender effect, there were 30 men (16 men with rheumatoid factor positive and 14 men with rheumatoid factor negative ) and 26 women (12 women with rheumatoid factor positive and 14 women with rheumatoid factor negative) (Table 1). 


\subsection{Age-Factor}

The onset of the disease occurs at all ages [20], but the incidence is highest between the ages of 35 and 50 years [21], this result was concordant with the finding of the current study. In the current study the age of patients range from (40-57).

There were no significant differences between different age categories regarding all studied parametersin $\mathrm{RF}$ negative group (Table 4) except for $\mathrm{Mn}(\mathrm{age}=55+, \mathrm{p}=0.005)$ and $\mathrm{Rb}$ ( age $=40-44, \mathrm{p}=0.044)$.

There were no significant differences between different age categories regarding all studied parametersin RF positive group (Table 5). Accordingly, there was no age effect.

It was reported that, the groups showed no significant age differences [22].

\subsection{Gender - Effacts}

There were no significant differences between RF positive and negative groups in males and females groups regarding all studied parameters (Tables 6,7) respectively.

\subsection{Correlation Coefficient}

There were no significant correlations among the studied parameters in RF negative group, except the correlation between $\mathrm{Mn}$ and age $(\mathrm{p}=0.013, \mathrm{r}=-0.472)$ (Table 8$)$.

There were no significant correlations among the studied parameters in RF positive group (Table 9).

These results give a good opportunity to the physicians to correct these TEs imbalance to improve the pathological status of the patients.

These variations are probably responsive to inflammation, OS and may be a part of autoimmune process in patients induced by cytokines and resulted from defensive mechanism of patients.

Accordingly, these results postulated that these TEs might play a role in pathophysiology of RA. These findings proved the possible association between altered levels of interested parameters with RA.

\section{Conclusion}

The variation of TEs in sera of patients with RA can enable us to high light on the role of TEs in both physiological and pathological status.

Since there is a significant reduction in serum levels of $\mathrm{Cs}$ and $\mathrm{Rb}$, so supplementation of these TEs could be important to get a benefit from TEs rebalance in serum, so supplementation with TEs could be necessary for RA therapy and even more important to individuals who are at high risk of developing RA.

The serum levels of $\mathrm{Co}, \mathrm{Mn}, \mathrm{Re}$ were significantly elevated in patients with RA as compared with the control group.

There were no significant differences in different age categories regarding interested parameters. There were no significant differences between RF positive and negative groups in males and females groups.
Further investigations are needed to study inununocytokines together with TEs and antioxidant enzymes activities in patients with RA.

Abbreviations: Co: Cobalt; Cs: cesium; IL: Interlukine; Mn: Manganese; OS: oxidative stress; RA: rheumatoid arthritis; Re: Rhenium; Rb: Rubedium; RF: rheumatoid factor; ROS: reactive oxygen species; TEs: Trace elements; TNF- $\alpha$ : Tumor necrosis factor- $\alpha$

\section{References}

[1] Symmons DP (2002). Epidemiology of rheumatoid arthritis: determinants of onset, persistence and outcome. Baillieres Best Pract Res ClinRheumatol, 16: 707-722.

[2] Ostrakhovitch EA, Afanas'ev IB. (2001). Oxidative stress in rheumatoid arthritis leukocytes: suppression by rutin and other antioxidants and chelators. Biochem. Pharmacol, 62: 743-746.

[3] Hitchon CA, Gabalawy HS EL (2004).Oxidation in rheumatoid arthritis Arthritis. Res. Ther., 6: 265-278.

[4] Pasha Q, Malik SA, Shaheen N, Shah MH.(2010). Investigation of trace metals in the blood plasma and scalp hair of gastrointestinal cancer patients in comparison with controls. Clin Chim Acta, 411: 531-539.

[5] Brig MN, Chatterje A and Shinde R, "text book medical biochemistry", 6th ed. Published by jitendarprij 2005, 178-557.

[6] Lovell DJ, Glass D, Ranz J, Kramer S, Huang B, Sierra RI, Henderson CJ, Passo M, Graham B, Bowyer S, Higgins G, Rennebohm R, Schikler KN, Giannini E.(2006). A randomized controlled trial of calcium supplementation to increase bone mineral density in children with juvenile rheumatoid arthritis. Arthrit Rheum, 54: 2235-2242.

[7] Arinola OG, Nwozo SO, Ajiboye JA, Oniye AH (2008c). Evaluation of trace elements and total antioxidant status in Nigerian cassava processors Pak. J. Nutr., 7(6): 770-772.

[8] Carl AB and Edward RA "Tietz Fundamentals of Clinical Chemistry", 6th Ed., Saunders an imprint of Elsevier 2008, 496507.

[9] Santamaria AB. (2003). "0Manganese Exposure, Essentiality and toxicity" Indian J. Medzres, 128: 484-500.

[10] Gordon RF (1977).Poultry Diseases. The English Language Book Society and Bailliere Tindall, London

[11] Chandra RK (1990). Micronutrients and immune functions: Anoverview. Annal New York Acad. Sci.587:9-16.

[12] De Carvalho PR, Gon Calves Pita MC, Loureiro JE, Tanaka HR and Ribeiro JS. (2010). "Manganese Deficiency in Bovines: connection between Manganese metalloenzyme Dependent in Gestation and Congenital Defects in New born Calves", Pakistan Journal of Nutrition, 9(5): 488-503.

[13] Yen, CK; Yano, Y; Budinger, TF; Friedland, RP; Derenzo, SE; Huesman, RH; O'Brien, HA.(1982). Brain tumor evaluation using Rb-82 and positron emission tomography. Journal of Nuclear Medicine, 23 (6): 532-537.

[14] Canavese, Caterina, Decostanzi, Ester, Branciforte, Lino, Caropreso, Antonio; Nonnato, Antonello; Sabbioni, Enrico (2001). Depression in dialysis patients: Rubidium supplementation before other drugs and encouragement. Kidney International. 60(3): 1201-1201. 
[15] William Niedermeier, James H. Griggs.(1971). Trace metal composition of synovial fluid and blood serum of patients with rheumatoid arthritis. Journal of Chronic Diseases, 23(8): 527-536.

[16] Zoli A, Altomaonte L, Carcchio R, Galossi A., Ruffini M.P. (1998). Serum Zinc, Copperin active rheumatoid arthritis: Correlation with interlukin $1 \beta$ and tumor necrosis factor- $\alpha$. Clin Rheumatol, 17(5):378-382.

[17] Mujtba GM Hashmi and Munir H Shah (2012). Comparative Assessment of Essential and Toxic Metals in the Blood of Rheumatoid Arthritis Patients and Healthy Subjects. Biol Trace Elem Res, 146(1):13-22. doi: 10.1007/s12011-0119220-9. Epub 2011 Oct 1

[18] Huda M. Ali and Mohammed A. Al-Zubaidi (2012). Evaluation of Trace Elements in Iraqi Patients with Rheumatoid Arthritis by using Atomic Absorption Spectrophotometer. Iraqi J Pharm Sci, 21(2):77-84.
[19] Satish KT and Reshu M. (2009).Assessment of mineral status ( $\mathrm{Zn}, \mathrm{Cu}, \mathrm{Mg}$ and $\mathrm{Mn}$ ) in Rheumatoid Arthritis patients in Chandigarh, India. Rheumatology Report, 1(5): 16-20.

[20] Krishna Mohan Surapneni and V S Chandrasada Gopan,(2008). Lipid Peroxidation and Antioxidant Status in Patients with Rhumatoid Anthritis. Indian Journal of Clinical Biochemistry, 23(1): 41-44.

[21] Prakash B. Desai S. Manjunath Sumangalakadi K. Chetana J. Vanishree, (2010). Oxidative stress and enzymatic antioxidant status in rheumatoid arthritis: a case control study. European Review for Medical and Pharmacological Sciences, 14 : 959967.

[22] Ala S, shokrzadeh M, Purshoja AM and Saravi S. (2009).Zinc and Copper plasma concentrations in Rheumatoid Arthritis patients from a selected population in Iran. Pakistan Journal of biological sciences, 12 (14): 1041-1044. 